\title{
Contributo alla conoscenza della biodiversità entomologica del Parco del Ticino: Cantharidae (Coleoptera, Elateroidea), Cleridae, Malachiidae e Dasytidae (Coleoptera, Cleroidea)
}

\author{
Gianfranco Liberti ${ }^{*}$, Danilo Mario Piccolino²
}

\begin{abstract}
A contribution to the knowledge of the insect biodiversity in the Ticino Valley Natural Park: Cantharidae (Coleoptera, Elateroidea), Cleridae, Malachiidae and Dasytidae (Coleoptera, Cleroidea).

The results of long-term entomological investigations carried out by the authors in the Ticino Valley Natural Park (Lombardy) on Cantharidae (Coleoptera, Elateroidea), Cleridae, Malachiidae and Dasytidae (Coleoptera, Cleroidea), as well as the study of material from several entomological collections, are here reported.

52 species are listed: 23 Cantharidae, 11 Malachiidae, 13 Dasytidae and 5 Cleridae. Most of them have been collected quite recently; however, some old observations have not been confirmed: e.g. Malthodes dispar and Malthodes recurvus were found more than 70 years ago only.
\end{abstract}

This contribution is a meaningful improvement to the current knowledge: Pilon (2002: 341-342) listed only 8 species belonging to the same families for the Ticino Valley Park.

After each species name, the following data have been added:

- main references useful for identification and/or distribution; tion;

- a short comment, mostly related to the known species distribu-

- a detailed list of localities including, in brackets, the year, the collector and - where applicable - the depository.

An asterisk indicates that the species also appears in Pilon (2002).

The Ticino Valley Park has a rich entomological fauna. In continental Italy approximately occur 170 Cantharidae, 75 Malachiidae, 80 Dasytidae and 30 Cleridae. That means about $15 \%$ of the Italian (excluding major islands) species have been found in the Park.

As a rough scheme 36 (ca. $69 \%$ ) out of the 52 listed species have a wide European (or Palaearctic, or cosmopolitan) distribution, $5(10 \%)$ are central European, 4 (8\%) Alpine and/or Apennine, 7 (13\%) Mediterranean.

The central European species are: Rhagonycha fuscitibia, Cratosilis laeta, Malthodes europaeus, Malthodes misellus, Aplocnemus integer.

The Alpine and/or Apennine species are: Malthodes cyphonurus, Ebaeus battonii, Danacea ambigua, Aplocnemus chalconatus.

The Mediterranean ones are: Malthodes recurvus, Axinotarsus ruficollis, Divales bipustulatus, Aplocnemus jejunus, Dasytes aeneiventris, Attalus minimus, Colotes maculatus.

The clear European composition of such fauna is also confirmed by the discovery of two interesting species: Rhagonycha testacea and

\footnotetext{
${ }^{1}$ Via Cascina Girola 81, 21040 Uboldo (VA), Italia

${ }^{2}$ Corso Torino 12, 27029 Vigevano (PV), Italia

E-mail: d.piccolino@alice.it

*Corresponding author: gianfranco.liberti@login.it

(C) 2014 Gianfranco Liberti, Danilo Mario Piccolino
}

Received: $8^{\text {th }}$ October 2014

Accepted for publication: $14^{\text {th }}$ November 2014
Malthodes europaeus. For $R$. testacea the Ticino Valley Park is seemingly the only sure Italian locality; whereas $M$. europaeus is also known from a few other sites in the central and western foothills of the Alps.

A comparison with the results of a faunistic survey at Bosco Fontana, a smaller natural reserve near Mantova, has also been attempted. Bosco Fontana and Ticino Park have a similar species composition concerning Cantharidae Cantharinae and Dasytidae, yet they meaningfully differ about Cantharidae Malthininae and Malachiidae.

Key-words: Coleoptera, Lombardy, Italy, records, faunistic, Ticino Park, checklist.

Riassunto - Vengono riportate le osservazioni effettuate dagli Autori nel Parco Lombardo della Valle del Ticino, riguardanti le famiglie Cantharidae (Coleoptera, Elateroidea), Cleridae, Malachiidae e Dasytidae (Coleoptera, Cleroidea), integrate coi dati provenienti da altre collezioni.

Sono elencate 52 specie: 23 Cantharidae, 11 Malachiidae, 13 Dasytidae e 5 Cleridae. Per ogni specie vengono riportate le segnalazioni disponibili per il Parco e informazioni sulla distribuzione generale. Per due specie, Malthodes dispar e Malthodes recurvus, gli unici dati disponibili risalgono ad oltre 70 anni fa.

Viene discussa la componente percentuale delle singole categorie corologiche nel territorio del Parco riferita alle famiglie in esame. Vengono infine confrontati i dati raccolti con quelli relativi alla Riserva Naturale di Bosco Fontana (Mantova).

Parole chiave: Coleoptera, Lombardia, Italia, segnalazioni, faunistica, Parco del Ticino, checklist.

\section{INTRODUZIONE}

Il presente contributo riassume i risultati di molti anni di ricerche entomologiche, svolte dagli Autori nel territorio del Parco del Ticino, mirate allo studio di quattro famiglie di Coleotteri: Cantharidae, Cleridae, Malachiidae e Dasytidae. Si tratta, in tutti i casi, d'insetti pollinivori o predatori di microartropodi, alati e buoni volatori, che vivono su fiori, erbe, arbusti e alberi. Le ricerche sono sempre state effettuate per "raccolta diretta", mediante "battitura" dei rami accessibili di alberi e arbusti e "sfalcio" dei prati con apposito retino.

Sono stati inoltre inclusi nel lavoro tutti i reperti noti agli autori, provenienti dal territorio dell'attuale Parco e più o meno recenti, conservati presso collezioni museali o private.

Sono state censite 23 specie di Cantharidae, 11 specie di Malachiidae, 13 specie di Dasytidae e 5 di Cleridae, per un totale di 52 specie, alcune delle quali osservate molti 
anni fa e mai più ritrovate (ad esempio Malthodes dispar e Malthodes recurvus, raccolti oltre 70 anni fa). Si tratta di un sostanziale ampliamento della conoscenza della fauna del Parco: nel Catalogo redatto 12 anni fa (Pilon, 2002: 341-342) venivano infatti citate, per Cantharidae, Cleridae, Malachiidae e Dasytidae rispettivamente 4, 2, 3 e 1 specie.

L'elenco delle località visitate dagli Autori è riportato in Appendice 1. La tassonomia segue il Catalogo Löbl \& Smetana (Kazantsev \& Brancucci, 2007; Löbl et al., 2007; Mayor, 2007).

\section{ELENCO COMMENTATO DELLE SPECIE OSSERVATE}

Famiglie e generi sono elencati in ordine sistematico, le specie sono in ordine alfabetico.

La bibliografia per specie si limita a pochi, o pochissimi lavori: sono stati indicati soltanto quelli giudicati più importanti ai fini del riconoscimento delle specie - così da render chiara l'interpretazione che gli autori hanno dato di ciascun taxon - e della individuazione degli areali di diffusione.

Dopo un breve commento, segue l'elenco delle località del Parco del Ticino ove la specie è stata osservata. Tali località sono ordinate da nord a sud. Accanto ad esse, escluse quelle tratte da Pilon (2002), sono riportati: l'anno di cattura, il nome del raccoglitore e la sigla che indica la collezione dove è conservato il materiale.

\section{Abbreviazioni}

Sigle che indicano le collezioni nelle quali è conservato il materiale:

$\mathrm{CAn}=$ Collezione Fernando Angelini (conservata presso il Museo di Storia Naturale di Firenze, sezione di Zoologia "La Specola")

$\mathrm{CBr}=$ Collezione Sergio Borroni, Legnano (MI)

$\mathrm{CCa}=$ Collezione Claudio Canepari, Milano

$\mathrm{CFr}=$ Collezione Mario Franciscolo (conservata presso il Museo di Genova)

$\mathrm{CLi}=$ Collezione Gianfranco Liberti, Uboldo (VA)

$\mathrm{CMt}=$ Collezione Fernando Montemurro, Taranto

$\mathrm{CPi}=$ Collezione Danilo Piccolino, Vigevano (PV)

$\mathrm{CSe}=$ Collezione Fabio Sereni, Milano

NHMB = Naturhistorisches Museum, Basel, Svizzera

(Coll. W. Wittmer)

MSNG = Museo Civico di Storia Naturale "Giacomo Doria", Genova

MSNM = Museo Civico di Storia Naturale di Milano

\section{Altre abbreviazioni}

* = specie già citate in Pilon (2002).

! = uno degli Autori (Liberti quando la collezione sia denominata $\mathrm{CLi}$, oppure Piccolino quando la sigla della collezione sia $\mathrm{CPi}$ ).

$?=$ raccoglitore ignoto.

CANTHARIDAE IMHOFF, 1856 (1815)

23 specie

Cantharis figurata Mannerheim, 1843

Horion, 1953: 26; Moscardini, 1968: 79; Dahlgren, 1979: 30.
Europa settentrionale e centrale, Caucaso, Siberia. In Italia la sua distribuzione resta da precisare: certamente presente al nord ove appare piuttosto comune. Fa parte di un gruppo di specie (almeno tre: pallida, cryptica e figurata) molto simili fra loro, la cui determinazione richiede dissezione ed esame dell'edeago (Dahlgren, 1979).

Riferiamo a questa specie la Cantharis rufa citata in Pilon (2002: 342).

MILANO: Nosate (2006, !, CLi); Ponte di Vigevano (2005, !, CLi). PAVIA: Vigevano (PILON 2002: 342); Zerbolò, Bosco Siro Negri (2009, !, CLi).

Cantharis fusca Linnaeus, 1758

Horion, 1953: 19; Moscardini, 1968: 55; Dahlgren, 1979: 25.

Ampia distribuzione Europea; comune in tutta Italia, escluse le Isole. Una sola località da segnalare all'interno del Parco.

PAVIA: Gambolò 7 km E (2014, !, CLi).

*Cantharis livida Linnaeus, 1758

Horion, 1953: 25; Moscardini, 1968: 69; Dahlgren, 1979: 24.

Ampia distribuzione europea e medio-orientale. Presente in tutta Italia, esclusa la Sardegna. Specie dal colore molto variabile, nel Parco del Ticino esiste la forma ad elitre nerastre.

VARESE: Castelnovate (2008, !, CLi).

PAVIA: Vigevano (PIlon 2002: 341); Gambolò 7 km E (2014, !, CLi); Vaccarizza (2011, !, CLi).

Cantharis pellucida Fabricius, 1792

Horion, 1953: 25; Moscardini, 1968: 66; Dahlgren, 1979: 24.

Europa settentrionale e centrale. Presente in tutta Italia, escluse le Isole. Comune al nord, meno frequente in Appennino.

MILANO: Castelletto di Cuggiono (2012, !, CLi).

PAVIA: Gambolò 7 km E (2014, !, CLi).

*Cantharis rustica Fallén, 1807

Horion, 1953: 20; Moscardini, 1968: 56; Dahlgren, 1979: 24.

Europa e Asia Minore. Presente in tutta Italia, escluse le Isole. Molto comune.

VARESE: Castelnovate (2009, !, CLi).

MILANO: Nosate (2006, !, CLi); Castelletto di Cuggiono (2012, !, CLi); Ponte di Vigevano (2005 e 2008, !, CLi).

PAVIA: Vigevano (Pilon 2002: 342); Pavia (Pilon 2002: 342).

Cratosilis laeta (Fabricius, 1792)

Porta, 1929: 62 (Pygidia); Horion, 1953: 45 (Pygidia); Moscardini, 1968: 88; Allenspach \& Wittmer, 1979: 52.

Rara sul versante settentrionale delle Alpi (Germania, Svizzera interna), comune nella Svizzera meridionale e molto comune in Italia settentrionale (Alpi e Pianura Padana). Presente sulle Alpi Marittime e sugli Appennini con una forma ad elitre scure. 
VARESE: Castelnovate (2008, !, CLi).

MILANO: Nosate (2006, !, CLi).

PAVIA: Gambolò 7 km E (2014, !, CLi); Bereguardo (2013, !, CLi); Zerbolò, Bosco Siro Negri (2009, !, CLi).

*Rhagonycha fulva (Scopoli, 1763)

Horion, 1953: 38; Moscardini, 1968: 85; Dahlgren, 1979: 35.

Europa, Caucaso, Siria. Tutta Italia, esclusa la Sardegna. Comunissima ovunque, a comparsa relativamente tardiva (giugno o luglio, a seconda dell'altitudine). La scarsità dei dati relativi al Parco del Ticino può non riflettere la sua reale abbondanza: le ricerche entomologiche nel Parco sono state effettuate, preferibilmente, nei mesi di aprile e maggio.

PAVIA: Vigevano (Pilon 2002: 342).

Rhagonycha fuscitibia Rey, 1891

Moscardini, 1968: 83 (R. femoralis); Dahlgren, 1976: 88 (R. improvisa); Dahlgren, 1979: 38 (R. improvisa).

= Rhagonycha improvisa Dahlgren, 1976 (posta in sinonimia da Kazantsev \& Brancucci, 2007: 263).

$=$ Rhagonycha nigritarsis auctt. (nec Brullé, 1832) (posizione sistematica chiarita da Dahlgren, 1976: 88).

Europa centro occidentale: Francia, Austria, Italia continentale e Sicilia (Moscardini, 1968: 84). Molto comune ovunque. Una delle specie più comuni del Parco. $R$. fuscitibia fa parte di un gruppo di specie molto simili fra loro, la cui determinazione può essere problematica.

VARESE: Castelnovate (2008, !, CLi).

MILANO: Nosate (2006, !, CLi); Castelletto di Cuggiono (2012, !, CLi); Bernate (2012, !, CLi); Casterno (2012, !, CLi); Ponte di Vigevano (2005, 2008 e 2011, !, CLi).

PAVIA: Sforzesca, Bosco Modrone (2005, !, CLi e CPi); Gambolò 7 km E (2014, !, CLi); Bereguardo (2013, !, CLi); Ponte di Bereguardo (2007, !, CLi); Zerbolò, Bosco Siro Negri (2009, !, CLi); Vaccarizza (2011 e 2013, !, CLi).

Rhagonycha lignosa (O. F. Müller, 1764)

Horion, 1953: 41; Moscardini, 1968: 87; Dahlgren, 1979: 36.

Tutta Europa, comune. Molto comune in tutta l'Italia peninsulare, presente in Sicilia (Moscardini, 1968: 87).

VARESE: Castelnovate (2008, !, CLi).

MILANO: Casterno (2012, !, CLi); Ponte di Vigevano (2005 e 2011, !, CLI).

PAVIA: Sforzesca: Bosco Modrone (2005, !, CLi); Gambolò 7 km E (2014, !, CLi); Ponte di Bereguardo (2007, !, CLi).

Rhagonycha lutea (O.F. Müller, 1764)

Horion, 1953: 38; Moscardini, 1968: 86; Dahlgren, 1979: 35.

Tutta Europa. Presente in tutta l'Italia continentale, comune. Nel Parco del Ticino sembra essere piuttosto comune solo in provincia di Pavia.

PAVIA: Sforzesca: Bosco Modrone (2005, !, CLi e CPi); Gambolò 7 km E (2014, !, CLi); Bereguardo (2013, !, CLi); Zerbolò, Bosco Siro Negri (2009, !, CLi).
Rhagonycha testacea Linné, 1758

Horion, 1953: 39; Moscardini, 1968: 93; Dahlgren, 1979: 36.

Europa centrale, Siberia. Per l'Italia vi sono diverse segnalazioni (Luigioni, 1929: 609; Porta, 1929: 59) tuttavia Moscardini (1968: 93) afferma di non avere mai visto esemplari italiani. Anche chi scrive non ha mai visto esemplari italiani di questa specie, ad eccezione dei pochi personalmente osservati nel Parco del Ticino.

MILANO: Castelletto di Cuggiono: $1 \widehat{\jmath}, 1$ ( 2012, !, CLi); Ponte di Vigevano: 2 우 $(2005$, !, CLi).

PAVIA: Zelata: 1 q $(1983$, !, CLi).

Malthinus balteatus Suffrian, 1851

Porta, 1929: 66; Horion, 1953: 58; Wittmer, 1974: 398; Wittmer, 1979: $41,43$.

Tutta Europa, Turchia. Tutta Italia peninsulare, Sicilia e Sardegna. Specie elusiva, mai comune. La sua presenza nel Parco pare limitata alla parte meridionale.

PAVIA: Gambolò 7 km E (2014, !, CLi); Ponte di Bereguardo (2007, !, CLi); Bereguardo (2013, !, CLi); Zerbolò, Bosco Siro Negri (2009,

!, CLi); Vaccarizza (2011 e 2013, !, CLi).

Malthinus flaveolus (Herbst, 1786)

Horion, 1953: 54; Wittmer, 1979: 41, 42; Allenspach \& Wittmer, 1979: 55.

Tutta Europa e Caucaso. In Italia solo al Settentrione (in Appennino è vicariato dall'affine $M$. deceptor Baudi, 1893): è comune sulle Alpi ma sembra essere alquanto raro nel Parco.

MILANO: Castano Primo (1963, !, CLi).

PAVIA: Sforzesca, Bosco Modrone (2005, !, CLi e CPi).

Malthinus glabellus Kiesenwetter, 1852

Horion, 1953: 59; Wittmer, 1974: 361, 387; Wittmer, 1979: 41, 42.

Europa centrale, tutta Italia peninsulare, dalle Alpi alla Calabria.

PAVIA: Sforzesca: Bosco Modrone (2005, !, CLi e CPi).

Malthinus seriepunctatus Kiesenwetter, 1851 (Fig. 1)

Horion, 1953: 55; Wittmer, 1974: 406; Wittmer, 1979: 41, 43.

Europa centrale e meridionale, tutta Italia peninsulare. Comune.

VARESE: Castelnovate (2008, !, CLi).

MILANO: Nosate (2006, !, CLi).

PAVIA: Sforzesca: Bosco Modrone (2005, !, CLi e CPi); Gambolò 7 km E (2014, !, CLi); Bereguardo (2013, !, CLi).

Malthodes cyphonurus Kiesenwetter, 1861

Kiesenwetter, 1872: pl. 5 fig. 57; Kiesenwetter, 1874: 60; Fiori, 1905: pl. 2 fig. 8; Fiori, 1906: 9; Liberti, 2015: in stampa.

Alpi centrali. In Italia Alpi e Prealpi piemontesi, lombarde, altoatesine e Pianura Padana adiacente. Nel Parco si trova solo nella sua parte più settentrionale.

VARESE: Castelnovate (2008, !, CLi). 
Malthodes dispar (Germar, 1824)

Liberti, 2011: 177.

Europa centro-occidentale, dalle Isole Britanniche all'Italia settentrionale; presente anche in Italia peninsulare e in Sicilia con forme poco studiate. La sua presenza nel Parco del Ticino, che consideriamo molto plausibile, dovrà essere confermata da osservazioni recenti.

MILANO: Turbigo (1935, Mariani, MSNM).

Malthodes europaeus Wittmer, 1970

Wittmer, 1970: 79, 81; Wittmer, 1979: 45; Liberti, 2015: in stampa.

Europa centrale, Penisola Balcanica settentrionale. In Italia presente su Alpi e Prealpi centrali e orientali, raro. Il ritrovamento nel Parco di questa interessante specie è limitata al settore più meridionale; sembra inoltre fosse più frequente nel secolo scorso: vi è una sola osservazione recente.

PAVIA: Ponte di Bereguardo (2007, !, CLi); Pavia (1961, !, CLi); Pavia: Santa Sofia (1930, Binaghi, NHMB); Cava Manara (1940, Mariani, MSNM); Confluenza Po-Ticino (1940 e 1941, Mariani, MSNM).

Malthodes marginatus (Latreille, 1806)

Horion, 1953: 61; Wittmer, 1979: 48; Liberti, 2015: in stampa.

Europa settentrionale e centrale. In Italia è presente nella Pianura Padana e in tutto l'Appennino, ove appare poco comune. Sembra mancare sulle Alpi.

VARESE: Castelnovate (2008, !, CLi).

MILANO: Castelletto di Cuggiono (2012, !, CLi); Bernate (2012, !,

CLi); Casterno (2012, !, CLi); Ponte di Vigevano (2008, !, CLi).

PAVIA: Ponte di Bereguardo (2007 e 2008, !, CLi).

Malthodes minimus (Linnaeus, 1758)

Horion, 1953: 67; Wittmer, 1979: 48; Liberti, 2015: in stampa.

Europa centrale e settentrionale, parte settentrionale della Penisola Balcanica. In Italia solo al nord: Pianura Padana e Appennino settentrionale, non comune. La sua presenza nel Parco del Ticino è stata documentata oltre 50 anni fa, ma non vi sono osservazioni recenti.

PAVIA: Pavia (1959, !, CLi).

Malthodes misellus Kiesenwetter, 1852

Wittmer, 1970: 79, 84; Wittmer, 1979: 46; Liberti, 2015: in stampa.

Europa centrale, Alpi. In Italia presente al nord e al centro. Presente, sebbene non comune, nel Parco del Ticino; è stato osservato anche nell'alto corso del fiume, a monte del Lago Maggiore, in territorio svizzero.

MILANO: Turbigo (1932, Mariani, MSNM); Castelletto di Cuggiono (2012, !, CLi); Bernate (2012, !, CLi); Ponte di Vigevano (2008, !, CLi).

Malthodes pumilus (Brébisson, 1835) Liberti, 2011: 197.

Specie molto piccola, le cui popolazioni sono apparentemente costituite da sole femmine: i maschi, almeno in
Italia, sono molto rari. Poiché la determinazione dei Malthodes si basa sui caratteri del maschio, l'identificazione di questa specie è problematica.

L'areale di Malthodes pumilus sembra essere molto esteso: tutta Europa, Turchia (Wittmer, 1970: 39), Iran (Delkeskamp, 1977: 406). In Italia pare essere presente ovunque, sulle Alpi sale oltre i $2.500 \mathrm{~m}$ ma, per la presenza quasi esclusivamente di femmine, non è certo si tratti sempre della stessa specie (Liberti, 2011: 198).

VARESE: Castelnovate (2008, !, CLi).

MILANO: Ponte di Vigevano (2005, !, CLi).

PAVIA: Gambolò 7 km E (2014, !, CLi).

Malthodes recurvus Baudi, 1871

Liberti 2011: 199

Specie distribuita in Francia sud orientale (Var, Alpes Maritimes) e in Italia nord occidentale (Piemonte meridionale, Lombardia meridionale, Liguria e provincia di Piacenza). Comune sui rilievi e raro in pianura. La sua presenza nel Parco del Ticino è stata documentata molti anni fa e, da allora, questa specie non è più stata osservata.

MILANO: Casterno (1941, Mariani, MSNM).

CLERIDAE LATREILLE, 1802

5 specie

*Thanasimus formicarius (Linné, 1758)

Horion, 1953: 152; Gerstmeier, 1998: 105.

Europa, nord Africa, Asia, nord America. Tutta Italia, non raro.

PAVIA: Vigevano (Pilon, 2002: 342).

Tilloidea unifasciata (Fabricius, 1787)

Horion, 1953: 148 (Tillus); Gerstmeier, 1998: 61.

Europa centrale e meridionale, incluso il sud dell'Inghilterra, assente nella Penisola Iberica. Tutta Italia ad eccezione della Sardegna. Piuttosto raro in tutto il suo areale.

MILANO: Ponte di Vigevano (2011, !, CLi).

*Trichodes apiarius (Linné, 1758)

Horion, 1953: 157; Gerstmeier, 1998: 151.

Europa centro-settentrionale e meridionale, nord Africa, Turchia, Caucaso, Asia centrale. Frequente in tutta Italia.

PAVIA: Vigevano (Pilon 2002: 342).

Necrobia rufipes (DeGeer, 1775)

Horion, 1953: 166; Gerstmeier, 1998: 213.

Specie cosmopolita, frequente in Italia. Venne raccolta, sebbene in minor numero, assieme alla specie seguente.

PAVIA: Pavia, 6 km SE (1958, !, CLi). 
Necrobia violacea (Linné, 1758) (Fig. 2)

Horion, 1953: 166; Gerstmeier, 1998: 213.

Specie cosmopolita, comune in Italia. Rinvenuta abbondante su substrato organico di origine animale parzialmente essiccato.

MILANO: Castano Primo (1965, !, CLi).

PAVIA: Pavia, 6 km SE (1958, !, CLi).

MALACHIIDAE FLEMING, 1821

10 specie

*Anthocomus (Celidus) equestris (Fabricius, 1781)

Horion, 1953: 114 (A. bipunctatus); Evers, 1979: 65 (A. bipunctatus); Mayor, 2007: 439.

= Telephorous bipunctatus Harrer, 1784 (sinonimia già riconosciuta da Abeille de Perrin, 1891: 193 nota).

Europa centrale e meridionale, Caucaso, Turchia, nord America. Solo in Italia settentrionale, piuttosto raro.

PAVIA: Vigevano (Pilon 2002: 342); Bereguardo (2013, Sereni, CSe).

Anthocomus (Anthocomus) rufus (Herbst, 1784)

Horion, 1953: 113 (A. coccineus); Evers, 1979: 65 (A. coccineus); Mayor, 2007: 439.

$=$ Cantharis coccinea Schaller, 1783 (nome non disponibile perché omonimo di Cantharis coccinea Linné, 1761, ora nel genere Pyrochroa)

Specie ad ampia distribuzione Euroturanica: tutta Europa, Turchia, Iran. Presente in tutta Italia, incluse le Isole. Specie palustre, si trova in autunno sulle spighette di Phragmites che crescono ai bordi di corpi d'acqua. La località sotto indicata è al di fuori dei confini del Parco del Ticino; tuttavia il Parco include la riva sud del Lago di Comabbio, a non più di $6 \mathrm{~km}$ di distanza dal Porto di Azzate, con ambiente lacustre molto simile.

VARESE: Porto di Azzate (2008, !, CLi)

*Axinotarsus ruficollis (Olivier, 1790)

Abeille de Perrin, 1891 (1890): 610; Horion, 1953: 98; Evers, 1979: 68; Mayor, 2007: 440.

Europa centrale e meridionale, nord Africa. Comune in tutta Italia, inclusa la Pianura Padana.

MILANO: Ponte di Vigevano (2005, !, CLi).

PAVIA: Vigevano (Pilon 2002: 342); Sforzesca, Bosco Modrone (2005, !, CLi e CPi); Gambolò 7 km E (2014, !, CLi); Zerbolò, Bosco Siro Negri $(2009$, !, CLi).

Clanoptilus elegans (Olivier, 1790)

Horion, 1953: 108 (Malachius); Evers, 1979: 63 (Malachius); Evers, 1985: 30, 35; Mayor, 2007: 443.

Europa occidentale, centrale e meridionale, Siberia. Molto comune in tutta l'Italia peninsulare.

MILANO: Nosate (2006, !, CLi).
Clanoptilus geniculatus (Germar, 1824)

Horion, 1953: 109 (Malachius); Evers, 1979: 63 (Malachius); Evers, 1985: 30, 35; Mayor, 2007: 443.

Europa, Caucaso, Turchia, Asia occidentale. Molto comune in tutta l'Italia peninsulare.

MILANO: Ponte di Vigevano (2011, !, CLi).

PAVIA: Zerbolò, Bosco Siro Negri (2009, !, CLi); Vaccarizza (2011, !, CLi).

Malachius aeneus (Linné, 1758)

Horion, 1953: 103; Evers, 1979: 62; Evers, 1985: 25, 32; Mayor, 2007: 448.

Specie ad ampia distribuzione euroasiatica, si trova anche in America del nord. Presente almeno in tutta l'Italia peninsulare. Non comune in Pianura Padana, gli unici reperti del Parco del Ticino risalgono a molti anni fa.

PAVIA: Pavia (1957, !, CLi).

Malachius australis Mulsant \& Rey, 1867

Evers, 1985: 25, 33; Mayor, 2007: 448.

Sud-est della Francia, Italia. Specie molto comune in tutta l'Italia peninsulare.

MILANO: Nosate (2006, !, CLi); Ponte di Vigevano (2008 e 2011, !, CLi).

PAVIA: Ponte di Bereguardo (2007, !, CLi); Zerbolò, Bosco Siro Negri (2009, !, CLi); Pavia (1959, !, CLi); Vaccarizza (2011 e 2013, !, CLi).

Attalus (Antholinus) minimus (Rossi, 1790)

Horion, 1953: 97; Evers, 1979: 66; Mayor, 2007: 420.

Specie del Mediterraneo centro-occidentale: Europa occidentale meridionale, nord Africa.

PAVIA: Ponte di Bereguardo (2007, !, CLi); Vaccarizza (2011, !, CLi).

Charopus concolor (Fabricius, 1801)

Abeille de Perrin, 1891 (1890): 252; Horion, 1953: 87; Evers, 1979: 59; Mayor, 2007: 441.

Europa centrale e meridionale. Segnalato di tutta Italia, frequente in Pianura Padana.

MILANO: Boffalora (1966, Spezia, CLi).

PAVIA: Vigevano (1961, !, CLi); Gambolò 7 km E (2014, !, CLi); Pavia (1959, !, CLi).

Colotes maculatus (Laporte de Castelnau, 1836) (Fig. 3) Horion, 1953: 83; Evers, 1979: 58; Mayor, 2007: 427.

Specie mediterranea che raggiunge l'Europa centrale. Comune in tutta Italia, ma piuttosto raro al nord.

PAVIA: Vigevano (1961, !, CLi); Gambolò 7 km E (2014, !, CLi); Vaccarizza (2013, !, CLi).

*Ebaeus battonii Pardo, 1962

Pardo Alcaide, 1962: 274; Evers, 1979: 67 (E. baltonii per "lapsus calami”); Mayor, 2007: 429. 
Italia peninsulare, Svizzera, Austria. Poco frequente in tutto il suo areale.

PAVIA: Vigevano (Pilon, 2002: 342).

\section{DASYTIDAE LAPORTE DE CASTELNAU, 1840} 13 specie

Danacea ambigua Mulsant \& Rey, 1868

Constantin, 2008: 208; Liberti, 2009b: 34; Constantin \& Liberti, 2011: 35,91 .

Francia (soprattutto nel meridione), Svizzera, tutta l'Italia continentale. Come quasi tutte le Danacea, anche D. ambigua si trova solo in collina e montagna. Per tale ragione nel Parco del Ticino si trova soltanto in provincia di Varese.

VARESE: Castelnovate (2008, !, CLi).

Dasytes (Mesodasytes) aeneiventris Küster, 1850

Liberti, 2004: 315; Liberti, 2009a: 359.

Mediterraneo occidentale e centrale: Europa meridionale dalla Spagna alla Grecia; nord Africa dal Marocco alla Tunisia. Le $q 0$ di Mesodasytes sono di determinazione incerta. Pertanto, la presenza nel Parco di questa specie, della quale sono state rinvenute solo 2 oq, è da confermare.

MILANO: Abbiategrasso: Cà di Biss (1923, Binaghi, MSNG). PAVIA: Zelata (1988, !, CLi).

Dasytes (Mesodasytes) aeratus Stephens, 1830 Liberti, 2004: 311; Constantin \& Liberti, 2011: 100.

Tutta Europa, Caucaso e Turchia. Molto comune in tutta l'Italia peninsulare, manca nelle isole.

VARESE: Casorate Sempione (2004, !, CLi).

MILANO: Castelletto di Cuggiono (2012, !, CLi); Casterno (1939, Mariani, MSNM; 2012, !, CLi); Abbiategrasso (2006, Diotti, CAn); Ponte di Vigevano (2005 e 2008, !, CLi).

PAVIA: Sforzesca (2006, Diotti, CAn); Gambolò: Bosco Giaretto (anno ignoto, !, CPi); Bereguardo (1936, Mariani, MSNM); Ponte di Bereguardo (2007, !, CLi); Vaccarizza (2011, !, CLi).

Dasytes (s. str.) niger (Linneo, 1761) (Fig. 4)

Liberti, 2004: 286; Constantin \& Liberti, 2011: 104.

Specie ad ampia distribuzione euroasiatica; in Europa solo al nord e al centro. In Italia è presente solo sulle Alpi e in Pianura Padana, dove è piuttosto raro.

MILANO: Turbigo (1923, ?, MSNG); Ponte di Vigevano (1960, Rossi, CLi; anno ignoto, !, CPi; 1987, 2005, 2008 e 2011, !, CLi); Morimondo $(1988, !$, CLi)

PAVIA: Gambolò 7 km E (2014, !, CLi).

Dasytes (Mesodasytes) plumbeus (Müller, 1776)

Liberti, 2004: 319; Constantin \& Liberti, 2011: 106.

Specie ad ampia distribuzione, dalla Spagna all'Iran e, probabilmente, alla Cina. In Europa è comune ovunque, anche se è meno abbondante o raro al nord. In Italia è comunissimo ovunque, ma manca in Sardegna (per contro è presente in Corsica). Fra i Dasytes è la specie più diffusa e abbondante nel Parco del Ticino.

VARESE: Sesto Calende (1959, ?, MSNM); Casorate Sempione (2004, !, CLi); Castelnovate (2008 e 2009, !, CLi).

MILANO: Castano Primo (1941, Mariani, MSNM; 1965, !, CLi); Nosate (2006, !, CLi); Ponte di Turbigo (1930, Binaghi, MSNG; 1933, Mariani, MSNM); Turbigo (anno ignoto, ?, MSNG; 1932, Mariani, MSNM; 2006, Borroni, CBr); Castelletto di Cuggiono (2012, !, CLi); Bernate (2012, !, CLi) Casterno (2012, !, CLi); Morimondo (1988, !, CLi); Ponte di Vigevano (2005, 2008 e 2011, !, CLi).

PAVIA: Vigevano (1940, Schatzmayr, MSNM; 1961, !, CLi); Sforzesca, Bosco Modrone (2005, !, CLi e CPi); Gambolò 7 km E (2014, !, CLi); Zelata (1983, !, CLi); Ponte di Bereguardo (2007, !, CLi); Bereguardo (1937, Mariani, MSNM; 2013, !, CLi); Zerbolò, Bosco Siro Negri (2009, !, CLi); Pavia (1961, !, CLi; 1982, Canepari, CCa); Cava Manara (1937, ?, MSNG); Confluenza Po - Ticino (1947, Bari, MSNG; 1963, Franciscolo, MSNG; 1966, Spezia, CLi); Vaccarizza (2011, !, CLi);.

Dasytes (Anthoxenus) subaeneus Schönherr, 1817 Liberti, 2004: 305; Constantin \& Liberti, 2011: 108.

Specie diffusa e relativamente comune in Europa centrale e meridionale. In Italia tende a diventare altitudinale: comune sulle Alpi Marittime e Cozie fino a oltre $2500 \mathrm{~m}$, raro (o assente) nelle Alpi centrali e presente, a quote inferiori, nel Carso Triestino in Venezia Giulia. Vive in quota anche in Appennino dall'Emilia alla Basilicata. Un unico reperto $(1$ o $)$ di quasi 50 anni fa nel Parco del Ticino.

MILANO: Boffalora (1966, Spezia, CLi).

Dasytes (Mesodasytes) virens (Marsham, 1802)

Liberti, 2004: 324; Constantin \& Liberti, 2011: 109.

Specie presumibilmente euroturanica, diffusa dalla Spagna almeno fino al Caucaso. In Italia si trova al nord e al centro, ma manca nelle regioni meridionali e nelle isole. Specie tendenzialmente legata ad aree montuose; manca, o è molto raro, in Pianura Padana. Di conseguenza, nel Parco del Ticino lo si trova soltanto in provincia di Varese.

VARESE: Castelnovate (2009, !, CLi).

*Divales bipustulatus (Fabricius, 1781)

Majer ,1984: 275; Constantin \& Liberti, 2011: 110.

Specie del Mediterraneo centrale il cui areale si estende dalle Alpi al nord Africa; presente anche nella Penisola Balcanica, in Bulgaria e in Grecia settentrionale. Relativamente comune nella Pianura Padana.

PAVIA: Vigevano (Pilon, 2002: 342).

Dolichosoma lineare (Rossi, 1792)

Liberti, 2009a: 364; Constantin \& Liberti, 2011: 40, 96.

Diffusione eurosibirica, in Europa dalla Finlandia alla Spagna. In Italia dalla Pianura Padana, ove è comune, al Lazio; in Italia meridionale è vicariata dall'affine Doli- 
chosoma simile. Presente anche in Sardegna. Comune nel Parco del Ticino.

MILANO: Ponte di Vigevano (2005 e 2011, !, CLi).

PAVIA: Vigevano (1961, !, CLi); Sforzesca, Bosco Modrone (2005, !, CLi e CPi); Gambolò 7 km E (2014, !, CLi); Bereguardo (1936, Binaghi, MSNG); Ponte di Bereguardo (2007, !, CLi); Zerbolò, Bosco Siro Negri (2009, !, CLi); Pavia (1959, !, CLi); Cava Manara (1937, Mariani, MSNM; 1947, ?, MSNG); Vaccarizza (2011, !, CLi).

Aplocnemus chalconatus (Germar, 1817)

Majer, 1982: 442; Liberti, 1994: 187; Constantin, 2007: 164.

Italia, Slovenia, Croazia. Comune in tutta l'Italia continentale ad eccezione del nord-ovest ove è sporadico; molto raro in Pianura Padana. Due soli vecchi reperti del Parco del Ticino.

PAVIA: Pavia (anno ignoto, Baudi, MSNG); Cava Manara (1937, ?, MSNG).
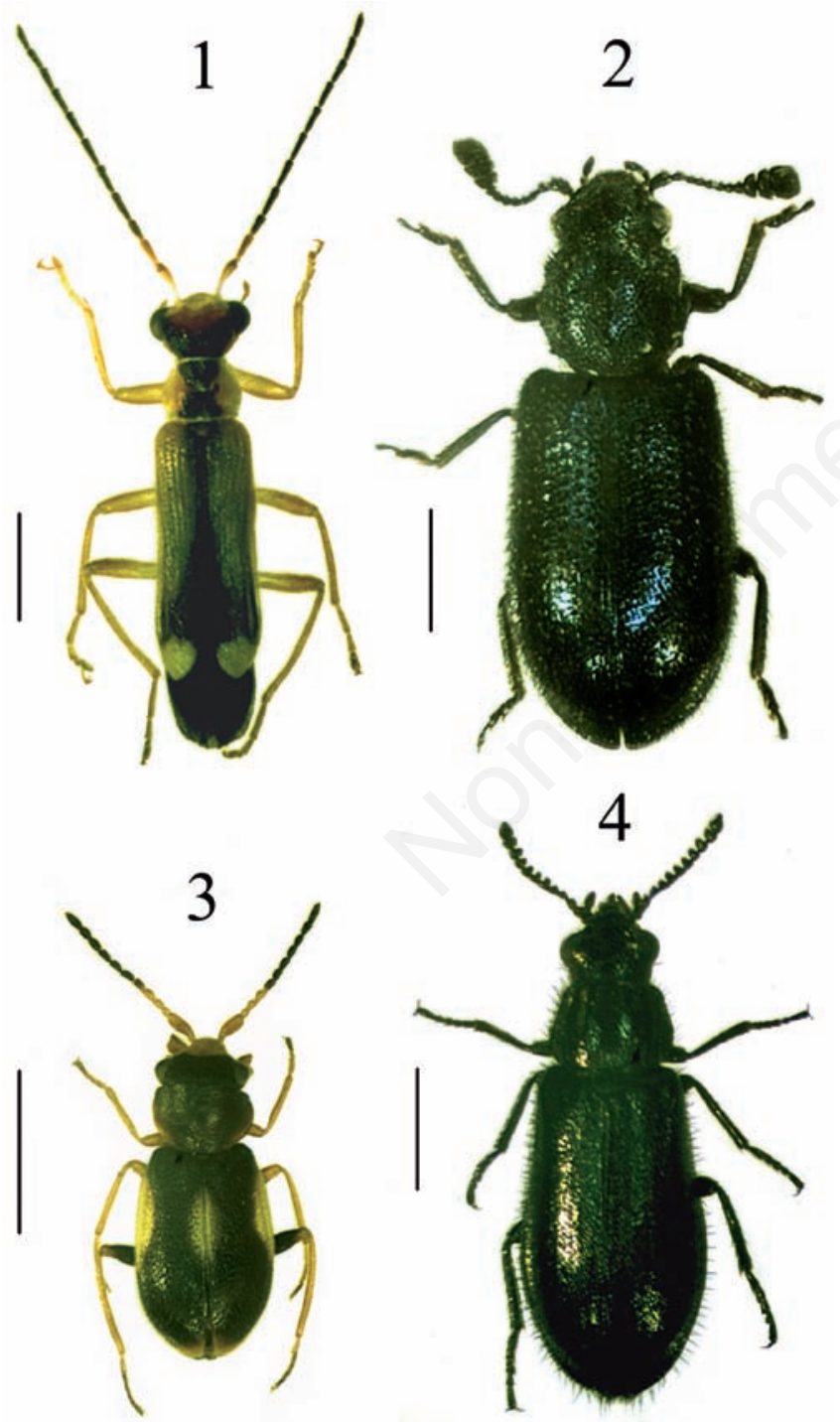

Figg. 1-4 - Habitus di alcune specie rinvenute nel Parco. 1) Malthinus seriepunctatus di Gambolò (7 km Est). 2) Necrobia violacea di Castano Primo. 3) Colotes maculatus di Gambolò (7 km Est). 4) Dasytes niger di Gambolò (7 km Est). Scala metrica $=1 \mathrm{~mm}$.
Aplocnemus integer Baudi, 1873

Majer, 1982: 437; Liberti, 1994: 182; Constantin, 2007: 162.

Italia, Ungheria, Bulgaria, Grecia settentrionale. Solitamente sporadico in tutta Italia Settentrionale, mancano dati relativi all'Italia Centrale; nel Meridione presente almeno in Basilicata e Calabria, ove sembra essere più comune. Piuttosto raro nella valle del Ticino, sebbene la località tipica sia Pavia, entro i limiti del Parco.

MILANO: Abbiategrasso (2004, Diotti, CAn); Ponte di Vigevano (2005, !, CLi).

PAVIA: Zerbolò: Bosco Siro Negri (2005, Diotti, CMt).

Aplocnemus jejunus Kiesenwetter, 1863

Liberti, 1994: 182; Constantin, 2007: 161; Liberti, 2009a: 369.

Rara specie ad ampia distribuzione approssimativamente centromediterranea: Italia (Isole incluse), Francia meridionale, Slovenia, Bulgaria, Grecia settentrionale. Molto raro in Pianura Padana.

VARESE: Castelnovate (2009, !, CLi).

Aplocnemus nigricornis (Fabricius, 1792)

Majer, 1982: 430; Liberti, 1994: 167; Constantin, 2007: 160.

Specie ad ampia diffusione europea. Non raro, ma infrequente, in tutta l'Italia continentale. Presente nella sola parte settentrionale del parco del Ticino.

VARESE: Coarezza (2004, !, CLi).

MILANO: Nosate (2006, !, CLi); Casterno (2012, !, CLi).

\section{DISCUSSIONE}

La fauna del Parco del Ticino si dimostra, come era da attendersi, particolarmente ricca. In Italia continentale vi sono circa 350 specie tra Cantharidae (circa 170), Malachiidae (circa 75), Dasytidae (circa 80) e Cleridae (meno di 30) (Audisio, Liberti et. al., 1995; Audisio, Gobbi et al., 1995). Questo significa che il Parco del Ticino ospita circa il $15 \%$ delle specie appartenenti alle famiglie in oggetto.

In queste quattro famiglie, su 52 specie documentate per il Parco, ben 36 (cioè oltre i 2/3: 69,2\%) sono ad ampia distribuzione: europea o euroasiatica, oppure cosmopolita. Delle rimanenti 16 specie ad areale più ridotto, $5(9,6 \%)$ possono essere considerate di tipo centroeuropeo, $4(7,7 \%)$ sono alpino-appenniniche e le rimanenti 7 $(13,5 \%)$ hanno carattere più nettamente mediterraneo:

Specie centro-europee con areale limitato: Rhagonycha fuscitibia, Cratosilis laeta, Malthodes europaeus, Malthodes misellus, Aplocnemus integer.

Specie alpino-appenniniche: Malthodes cyphonurus, Ebaeus battonii, Danacea ambigua, Aplocnemus chalconatus.

Specie mediterranee: Malthodes recurvus, Axinotarsus ruficollis, Divales bipustulatus, Aplocnemus jejunus, Dasytes aeneiventris, Attalus minimus, Colotes maculatus.

Quindi, quasi l' $80 \%$ delle specie rinvenute nel Parco hanno distribuzione europea più o meno ampia (o sono 
cosmopolite), meno dell' $8 \%$ sono a distribuzione alpinoappenninica e meno del $15 \%$ hanno carattere mediterraneo.

Il carattere spiccatamente europeo di questa fauna è ulteriormente confermato dalla presenza di almeno due specie significative: Rhagonycha testacea e Malthodes europaeus, per le quali il Parco del Ticino costituisce uno dei pochi biotopi italiani ove la loro presenza appare certa.

Il Parco è, in Pianura Padana, una fra le aree di maggior estensione ove il territorio sia soggetto ad azione antropica relativamente meno pesante. Un altro sito pa- dano di interesse naturalistico, assai più piccolo, è Bosco della Fontana, presso Mantova (Mason et al., 2002). Uno studio lì condotto (Hardersen et al., 2014), basato su prelievi mediante trappole "Malaise", ha evidenziato la presenza di 13 specie di Cantharidae, 3 di Malachiidae e 4 di Dasytidae. La minor ricchezza faunistica è certamente legata alla minore estensione e alla maggiore uniformità dell'ambiente, mentre il Parco del Tícino è più grande e diversificato. Fra le specie del Parco del Ticino e quelle di Bosco della Fontana vi è ampia sovrapposizione; tuttavia è interessante notare come, in alcuni gruppi sistematici, vi siano anche notevoli differenze.

Tab. 1 - Comparazione faunistica tra le riserve naturali del Parco del Ticino e del Bosco Fontana.

\begin{tabular}{|l|l|l|l|l|}
\hline Gruppo sistematico & $\begin{array}{l}\text { Specie presenti } \\
\text { nel Parco del } \\
\text { Ticino }\end{array}$ & $\begin{array}{l}\text { Specie presenti a } \\
\text { Bosco Fontana }\end{array}$ & $\begin{array}{l}\text { Numero di } \\
\text { specie comuni ad } \\
\text { entrambi i territori }\end{array}$ & $\begin{array}{l}\text { Specie di Bosco } \\
\text { Fontana non reperite } \\
\text { nel Parco del Ticino }\end{array}$ \\
\hline Cantharidae Cantharinae & 11 & 5 & 5 & nessuna \\
\hline Cantharidae Malthininae & $\begin{array}{l}12 \\
\text { di cui } \\
4 \text { Malthinus } \\
8 \text { Malthodes }\end{array}$ & $\begin{array}{l}8 \\
\text { di cui } \\
6 \text { Malthinus }\end{array}$ & 4 & $\begin{array}{l}\text { Malthodes facetus } \\
\text { Malthodes lobatus } \\
\text { Malthodes siculus } \\
\text { Malthodes umbrosus }\end{array}$ \\
\hline Malachiidae & 11 & 3 & nessuna & $\begin{array}{l}\text { Sphinginus lobatus } \\
\text { Troglops albicans } \\
\text { Troglops silo }\end{array}$ \\
\hline Dasytidae & 13 & 4 & 4 & nessuna \\
\hline
\end{tabular}

Da Tab. 1 appare come le minori sovrapposizioni si verifichino nel genere Malthodes e nella famiglia Malachiidae. Attribuire tali differenze soltanto a difetto di ricerca (o alle diverse modalità di ricerca) potrebbe essere semplicistico, anche perché in alcuni gruppi la sovrapposizione è completa. Nel caso dei Malthodes, ben 3 della 4 specie presenti a Bosco Fontana, ma assenti nel Parco del Ticino (M. facetus, M. siculus e M. umbrosus), hanno diffusione prevalentemente Appenninica. Nel caso dei Malachiidae tutte e tre le specie hanno areale Europeo Meridionale (sebbene Troglops albicans meno spiccatamente delle altre due).

Entrambi questi biotopi possono essere considerati come "isole" circondate da un "mare" agricolo e molto antropizzato. Le differenze riscontrate possono avere diverse spiegazioni: la continuità boschiva della Val Padana potrebbe essere stata, anche in tempi relativamente recenti, assai diversa da quella attuale, $o$, più semplicemente, le specie incluse nelle Cantharinae e nei Dasytidae potrebbero essere dotate di maggiore mobilità e capacità di spostamento su lunghi percorsi rispetto ai Malthodes e ai Malachiidae. Purtroppo però i dati disponibili sono al momento insufficienti per poter approfondire l'argomento.

\section{Ringraziamenti}

Claudio Peja, Adriano Bellani e Valentina Parco, della Direzione del Parco Lombardo della Valle del Ticino, con la loro grande pazienza e preziosa collaborazione, ci hanno permesso la preparazione e la pubblicazione del presente lavoro.

Fabrizio Rigato, Maurizio Pavesi e Michele Zilioli del Museo Civico di Storia Naturale di Milano; Roberto Poggi, del Museo Civico di Storia Naturale "Giacomo Doria" di Genova e Matthias Borer del Naturhistorisches Museum, Basel ci hanno facilitato, in ogni modo, l'accesso alle collezioni delle rispettive Istituzioni Museali.

Fernando Angelini, Sergio Borroni, Claudio Canepari, Fernando Montemurro e Fabio Sereni ci hanno gentilmente consentito lo studio delle loro collezioni.

Fabrizio Rigato ha rivisto il testo del lavoro, apportando un rilevante contributo al suo miglioramento.

\section{BIBLIOGRAFIA}

Abeille de Perrin E., 1891 - Malachiidae. Malachides d'Europe et pays voisins. Annales de la Société Entomologique de France [pubblicato in più riprese] 1890: 181-260, 331-420, 567-680; 1891: 115-230, 405-446 e tavole 6, 7. 
Allenspach V. \& Wittmer W., 1979 - Insecta Helvetica Catalogus. 4. Coleoptera. Cantharoidea, Cleroidea, Lymexylonoidea. Schweizerischen Entomologischen Gesellschaft.

Audisio P., Liberti G., Nardi G. \& Poggi R., 1995 - Coleoptera Polyphaga VIII (Cantharoidea, Dermestoidea). In: Checklist delle specie della fauna italiana 53. Minelli A., Ruffo S. \& La Posta S. (eds.). Calderini, Bologna.

Audisio P., Gobbi G., Liberti G. \& Nardi G., 1995 - Coleoptera Polyphaga IX (Bostrichoidea, Cleroidea, Lymexyloidea). In: Checklist delle specie della fauna italiana 54. Minelli A., Ruffo S. \& La Posta S. (eds.). Calderini, Bologna.

Constantin R., 2007 - Révision des Aplocnemus de France avec description de trois nouvelles espèces. Observations taxonomiques et faunistiques sur les espèces françaises de Dasytidae et Acanthocnemidae (Coleoptera, Cleroidea). Bulletin de la Société entomologique de France, 112 (2): 151-170.

Constantin R., 2008 - Révision des Danacea Laporte de Castelnau, 1836, de France continentale et de la péninsule Ibérique avec description de deux espèces nouvelles. Les espèces a soies prothoraciques antéversées (Coleoptera, Dasytidae). Bulletin de la Société entomologique de France, 113 (2): 207-225.

Constantin R. \& Liberti G., 2011 - Coléoptères Dasytidae de France. Musée des Confluences, Lyon.

Dahlgren G., 1976 - Zur Taxonomie der Gattungen Rhagonycha und Cantharis (Col. Cantharidae). Mitteilungen Entomologische Gesellschaft Basel (N.F.): 26: 88-90.

Dahlgren G., 1979 - Cantharidae (ohne Tribus Malthinini). In: Die Käfer Mitteleuropas, Band 6, Diversicornia. Freude H., Harde K. W. \& Lohse G. A. (eds.). Goecke \& Evers Verlag, Krefeld: 19-39.

Delkeskamp K., 1977 - Pars 165 (I): Cantharidae - Coleopterorum Catalogus Supplementa edita a J. A. Wilcox. Dr. W. Junk bv Publishers, The Hague.

Evers A. M., 1979 - 29. Familie: Malachiidae. In: Die Käfer Mitteleuropas, Band 6, Diversicornia. Freude H., Harde K. W. \& Lohse G. A. (eds.). Goecke \& Evers Verlag, Krefeld: 53-69.

Evers A. M., 1985 - Aufteilung der paläarktischen Arten des Gattungscomplexes Malachius F. 54. Beitrag zur Kenntnis der Malachiidae. Entomologische Blätter für Biologie und Systematik der Käfer, 81 (1-2): 1-40.

Fiori A., 1905 - Revisione delle specie italiane a me note del genere Malthodes Kiesw. (con due tavole). Rivista Coleotterologica Italiana, 3 (12): 221-253.

Fiori A., 1906 - Revisione delle specie italiane a me note del genere Malthodes Kiesw. (cont. e fine, ved. N. 12, anno 1905). Rivista Coleotterologica Italiana, 4 (1): $1-26$.

Gerstmeier R., 1998 - Checkered beetles. Illustrated key to the Cleridae and Thanerocleridae of the western Palaearctic. Margraf Verlag, Weikersheim.

Hardersen S., Curletti G., Leseigneur L., Platia G., Liberti G., Leo P., Cornacchia P. \& Gatti E., 2014 - Spatiotemporal analysis of beetles from the canopy and ground layer in an italian lowland forest. Bulletin of Insectology, 67 (1): 87-97.
Horion, A., 1953 - Faunistik der mitteleuropäischen Käfer. Band III: Malacodermata, Sternoxia (Elateridae bis Throscidae). Entomologische Arbeiten aus dem Museum G. Frey, Sonderband, München.

Kazantsev S. \& Brancucci M., 2007 - Family Cantharidae Imhoff, 1856 (1815). In: Catalogue of Palaearctic Coleoptera, Volume 4. Elateroidea. Derodontoidea. Bostrichoidea. Lymexyloidea. Cleroidea. Cucujoidea. Löbl I. \& Smetana A. (eds.). Apollo Books, Stenstrup: 234-298.

Kiesenwetter H. von, 1872 - Revision der europäischen Arten der Gattung Malthodes (Erstes Stück). Berliner entomologische Zeitschrift, 16: 369-392.

Kiesenwetter H. von, 1874 - Revision der europäischen Arten der Gattung Malthodes (Zweites Stück). Berliner entomologische Zeitschrift, 18: 45-70.

Liberti G., 1994 - Revisione delle specie italiane del genere Aplocnemus Stephens (Coleoptera Melyridae Rhadalinae. Memorie della Società entomologica italiana, Genova, 73: 153-194.

Liberti G., 2004 - Il genere Dasytes Paykull in Italia. Revisione e catalogo topografico, sinonimico e bibliografico delle specie italiane. (Coleoptera, Dasytidae). Annali del Museo Civico di Storia Naturale "Giacomo Doria", Genova, 96: 253-340.

Liberti G., 2009a - The Dasytidae (Coleoptera) of Sardinia. Zootaxa, 2318: 339-385.

Liberti G., 2009b - The Danacea of the Balkan Peninsula. A revision. Memorie della Società entomologica italiana, Genova, 88: 17-116.

Liberti G., 2011 - Le specie di Malthodes Kiesenwetter, 1852 delle Alpi Marittime e Liguri (Coleoptera, Cantharidae). Annali del Museo Civico di Storia Naturale "Giacomo Doria", Genova, 103: 147246.

Liberti G., in stampa - I Malthodes del nord-ovest d'Italia (Col., Cantharidae) (Malthodes di Lombardia: $2^{\circ}$ contributo alla conoscenza dei Malthodes italiani). Annali del Museo Civico di Storia Naturale "Giacomo Doria", Genova.

Löbl I., Rolcik J., Kolibac J., \& Gerstmeier R., 2007 Family Cleridae Latreille, 1802. In: Catalogue of Palaearctic Coleoptera. Volume 4. Elateroidea. Derodontoidea. Bostrichoidea. Lymexyloidea. Cleroidea. $\mathrm{Cu}-$ cujoidea. Löbl I. \& Smetana A. (eds.). Apollo Books, Stenstrup: 367-384.

Luigioni P., 1929 - I Coleotteri d'Italia. Catalogo sinonimico-topografico-bibliografico. Memorie della Pontificia Accademia delle Scienze Nuovi Lincei, Roma (Serie II), 13: 1-1159.

Majer K., 1982 - Species of the genus Aplocnemus of Middle Europe. Deutsche entomologische Zeitschrift N. F., 29 (4-5): 421-445.

Majer K., 1984 - A revision of the genus Divales Cast. (Coleoptera, Melyridae, Dasytinae). Entomologica Basiliensia, 9: 265-317.

Mason F., Cerretti P., Tagliapietra A., Speight M. C. D. \& Zapparoli M., 2002 - Invertebrati di una foresta della Pianura Padana. Bosco della Fontana. Primo contributo. Conservazione Habitat Invertebrati 1. Gianluigi Arcari Editore, Mantova. 
Mayor A., 2007 - Family Dasytidae Laporte, 1840. Family Malachiidae Fleming, 1821: 415-454. In: Catalogue of Palaearctic Coleoptera. Volume 4. Elateroidea. Derodontoidea. Bostrichoidea. Lymexyloidea. Cleroidea. Cucujoidea. Löbl I. \& Smetana A. (eds.). Apollo Books, Stenstrup: 388-415.

Moscardini C., 1968 - Ricerche sulla fauna appenninica XCVIII. I coleotteri cantaridini della regione appenninica (Coleoptera-Cantharidae). Memorie del Museo Civico di Storia Naturale di Verona, 16: 49-96.

Pardo Alcaide A., 1962 - Malachiidae del Meditarraneo occidental (Coleoptera). Eos, Revista Española de Entomologia, 38: 263-298.

Pilon N., 2002 - Coleotteri. In: Atlante della biodiversità nel Parco del Ticino. Volume 1. Elenchi Sistematici. Furlanetto D. (ed.). Ed. Consorzio Lombardo Parco della Valle del Ticino, Pontevecchio di Magenta (MI): 311-364.

Porta A., 1929 - Fauna Coleopterorum Italica. Vol III: Diversicornia. Stabilimento Tipografico Piacentino, Piacenza.

Wittmer W., 1974 - Zur Kenntnis der Gattung Malthinus Latr. II (Col., Cantharidae) (60. Beitrag zur Kenntnis der Paläarktischen Cantharidae). Entomologische Arbeiten aus dem Museum G. Frey, München, 25: 358427.

Wittmer W., 1979 - 4. Tribus Malthinini. In: Die Käfer Mitteleuropas, Band 6, Diversicornia. Freude H., Harde K. W. \& Lohse G. A. (eds.). Goecke \& Evers Verlag, Krefeld: 40-51.

\section{APPENDICE 1}

Sono qui riportate le località visitate dagli Autori dopo il 2004. In tutti i casi le osservazioni sono state effettuate su erbe, alberi e arbusti mediante "retino da sfalcio" e "ombrello giapponese".

Le località sono elencate da nord a sud.

Nel testo sono riportate ulteriori località (per le quali non sono disponibili informazioni aggiuntive) ricavate dai cartellini applicati agli esemplari di Musei o collezioni private.

\section{Provincia di Varese}

Porto di Azzate

sul Lago di Varese, fra Bodio e Capolago, 20.X.2008 (ricerca sulle spighette di Phragmites)

Coarezza

$1 \mathrm{~km}$ E (strada proveniente da Somma Lombardo), 28.IV.2004

Casorate Sempione

ca. $2 \mathrm{~km}$ a W del paese, 28.IV.2004

Castelnovate nei pressi della "Pista Prova Pneumatici": 9.IV.2009: $45^{\circ} 37.6^{\prime} \mathrm{N} 08^{\circ} 40.0^{\prime} \mathrm{E}$ 22.IV.2009: $45^{\circ} 37.2^{\prime} \mathrm{N} 08^{\circ} 40.4^{\prime} \mathrm{E}$

\section{Provincia di Milano}

Nosate boschi sulla riva sx del fiume, fra Nosate e Turbigo, 10.V.2006

Castelletto di Cuggiono 27.IV.2012: $45^{\circ} 29.2^{\prime} \mathrm{N} 08^{\circ} 47.3^{\prime} \mathrm{E}$

Bernate Ticino nei pressi del ponte Autostrada A4, 28.IV.2012 $45^{\circ} 28.1^{\prime} \mathrm{N} 08^{\circ} 47.7^{\prime} \mathrm{E}$

Casterno 28.IV.2012, $45^{\circ} 25.3^{\prime} \mathrm{N} 08^{\circ} 49.9^{\prime} \mathrm{E}$

Ponte di Vigevano riva sinistra del Ticino (lato Abbiategrasso) 13.V.2005 27.IV.2011: $45^{\circ} 20.4^{\prime} \mathrm{N} 08^{\circ} 53.5^{\prime} \mathrm{E}$

\section{Provincia di Pavia}

Sforzesca

Bosco Modrone (oltre la Fattoria Ronchi), 28.V.2005

Gambolò

7 km E (nei pressi della Cascina Portalupa), 10.V.2014

Ponte di Bereguardo riva destra del Ticino, a monte del ponte, 20.IV.2007

Bereguardo a valle del ponte, riva sinistra del Ticino, 11.V.2013 45⒔9'N $09^{\circ} 01.6^{\prime} \mathrm{E}$

Zerbolò

ca. 2 km E, Bosco Siro Negri, 24.V.2009 $45^{\circ} 12.5^{\prime} \mathrm{N} 09^{\circ} 03.5^{\prime} \mathrm{E}$

Vaccarizza

4.V.2011: $45^{\circ} 08.9^{\prime} \mathrm{N} 09^{\circ} 14.4^{\prime} \mathrm{E}$ 11.V.2013: $45^{\circ} 08.8^{\prime} \mathrm{N} 09^{\circ} 14.7^{\prime} \mathrm{E}$ 